\title{
Intracellular Protein Determination using Droplet-Based
}

\section{Immunoassays}

Chiara Martino ${ }^{1}$, Michele Zagnoni ${ }^{1}{ }^{*}$, Mairi E. Sandison ${ }^{2}$, Mayuree Chanasakulniyom ${ }^{1}$, Andrew R. Pitt ${ }^{2}$ and Jonathan M. Cooper ${ }^{1 *}$

${ }^{1}$ Division of Biomedical Engineering, School of Engineering, University of Glasgow, Glasgow, G12 8LT, UK

${ }^{2}$ Institute of Molecular, Cell and Systems Biology, College of Medical, Veterinary and Life Sciences, University of Glasgow, Glasgow, G12 8QQ, UK

* michele.zagnoni@eee.strath.ac.uk, j.cooper@elec.gla.ac.uk

RECEIVED DATE (to be automatically inserted after your manuscript is accepted if required according to the journal that you are submitting your paper to)

CORRESPONDING AUTHOR FOOTNOTE. Michele Zagnoni, michele.zagnoni@eee.strath.ac.uk, current address: Centre of Microsystems and Photonics, Dept. Electron. Electric. Eng., University of Strathclyde, Glasgow, G1 1XW, UK. Jonathan M. Cooper, j.cooper@elec.gla.ac.uk, Division of Biomedical Engineering, School of Engineering, University of Glasgow, Glasgow, G12 8LT, UK.

ABSTRACT: This paper describes the implementation of a sensitive, on-chip immunoassay for the analysis of intracellular proteins, developed using microdroplet technology. The system offers a number of analytical functionalities, enabling the lysis of low cell numbers, as well as protein detection and quantification, integrated within a single process flow. Cells were introduced into the device in 
suspension and were irreversibly electroporated in situ. The cell lysate was subsequently encapsulated together with antibody-functionalised beads into stable, water-in-oil droplets, which were stored onchip. The binding of intracellular proteins to the beads was monitored fluorescently. By analysing many individual droplets and quantifying the data obtained against standard additions, we measured the level of two intracellular proteins, namely HRas-mCitrine, expressed within HEK-293 cells, and actin-EGFP, expressed within MCF-7 cells. We determined the concentrations of these proteins over five orders of magnitude, from $\sim 50 \mathrm{pM}$ to $1 \mu \mathrm{M}$. The results from this semi-automated method were compared to those for determinations made using Western blots, and were found not only to be faster, but required a smaller number of cells.

KEYWORDS: microdroplets, electroporation, lab-on-a-chip, immunoassay.

\section{Introduction}

Although conventional laboratory procedures for protein analysis, including Western blot methods ${ }^{1}$ and enzyme-linked immunosorbent assays (ELISA) ${ }^{2}$, enable sensitive detection and quantification of proteins, they are often labour-intensive, time-consuming and can require large sample volumes (i.e. $\mathrm{mL}$ ), especially when multiplexed. Microarray technologies and bead-based techniques can also be used to perform sensitive, multiplexed immunoassays ${ }^{3-7}$, and these technologies have already resulted in the development of methods for enriching and quantifying cellular proteins, with applications in diagnostics and proteomics, such as the elucidation of intracellular signalling pathways and cell to cell communication $^{8-10}$.

In the last two decades, considerable effort has been invested in the development of lab-on-a-chip (LOC) technologies ${ }^{11-14}$, using greatly reduced sample volumes and enabling faster and more sensitive, multiplexed analysis to address a wide variety of biological problems. A number of LOC systems using antibody-conjugated, bead-based methods have previously been described, including those using microfluidic trapping ${ }^{15-18}$ and micro-flow cytometry ${ }^{19-21}$. Epifluorescence microscopy has also been 
combined with multi-step, on-chip processing to create methods for multiplexed analysis using populations of encoded beads ${ }^{22}$.

Of the microfluidic techniques available for manipulating low volumes, as required for single cell analysis, emulsion-based microfluidics perhaps holds the greatest potential. This technology, also known as droplet microfluidics, has been successfully used to produce assays with volumes as low as several 10 s of fL for a variety of chemical and biological applications ${ }^{23}{ }^{24}$. Droplet microfluidic systems form highly monodisperse emulsions ${ }^{25}$ (e.g. water droplets in oil, W/O) at up to $\mathrm{kHz}$ frequencies, with droplet diameters in the micrometre range, offering the potential for multiplexed, high-throughput analysis. Each W/O droplet can constitute an isolated and biocompatible microcompartment, suitable for carrying out quantitative cell-based studies, or low volume DNA and protein assays.

Droplet microfluidic systems for protein analysis have been reported in the literature, including a system for performing immunoassays using encapsulated single cells in W/O droplets, where the cells were lysed by laser photolysis ${ }^{26}$. Although suitable for performing enzymatic activity assays at the single-cell level, this approach is not suitable for higher-throughput applications. Cells have also been trapped within droplets for the detection of surface protein biomarkers using enzymatic amplification ${ }^{27}$ and a segmented gas-liquid flow microsystem has been reported for performing stimulation of mammalian cells followed by chemical cell lysis ${ }^{28}$. However, fully integrated analysis has not been demonstrated in either case, with the antibody staining and flow cytometry measurement steps being performed down stream and off-chip.

In seeking to validate new LOC methodologies for protein determination, we present here a droplet microfluidic architecture that enables rapid intracellular protein quantification from cell suspensions, using low cell numbers, reduced sample volumes, developed within the framework of a fully integrated, on-chip procedure. By combining compartmentalisation with high-flow rates and on-chip electrical lysis, epifluorescence protein detection was performed on numerous microdroplets, inside which cell lysate and antibody-conjugated beads had been encapsulated. 
On-chip electrical lysis is an attractive technique for chip based technologies, due to the simplicity of integration, the lack of interference arising from the addition of detergents (as is the case with chemical lysis of cells), and its high efficiency. Thus, a suspension containing less than a thousand cells can be injected into the device and cell lysis can be performed using integrated microelectrodes, prior to droplet formation, without any need for further sample preparation.

Results showed that the system has a detection range spanning from tens of $\mathrm{pM}$ to $\mu \mathrm{M}$, with total analysis times typically within the order of two hours. Results obtained from the on-chip immunoassay were at least comparable to those acquired using conventional procedures, with the microfluidic dropletbased system enriching and quantifying intracellular proteins in a fully integrated manner using reduced cell numbers. In combination with existing bead coding technologies ${ }^{22}$, where a library of identifiable beads each functionalised with a different capture antibody can be created, this droplet-based approach could lead to a system for rapid, multiplexed detection.

\section{Materials and Methods}

\section{Materials}

Polydimethylsiloxane (PDMS) (Sylgard 184 Silicone Elastomer, Dow Corning) and glass microscope slides were used for device fabrication. Fluorocarbon oil (Fluorinert FC40, 3M) containing 2\% wt PEGPFPE blockcopolymer (Raindance Technologies, Inc) was used as the continuous phase. The dispersed aqueous phase was either phosphate buffer saline (PBS) containing an appropriate antibody concentration and $1 \% \mathrm{v} / \mathrm{v}$ bovine serum albumin (BSA), a cell lysate solution or a suspension of cells in 0.6M D-Sorbitol. G-actin buffer contained $5 \mathrm{mM}$ Tris $\mathrm{pH} 8.0,0.2 \mathrm{mM}$ calcium chloride, $0.2 \mathrm{mM}$ ATP and $0.5 \mathrm{mM}$ DL-Dithiothreitol. Further details of the materials used are provided in Supporting Information.

\section{Device fabrication}

Microelectrodes were fabricated on a glass substrate, using standard photolithography, metal evaporation and lift-off techniques, and were passivated with a 60nm layer of hydrogen silsesquioxane. 
Microchannels were fabricated in PDMS using standard soft lithography techniques. The PDMS channel and electrode-patterned substrate were irreversibly bonded together by oxygen plasma bonding and the resulting microchannel was coated with Aquapel (PPG Industries, PA, USA). Details of the device fabrication protocols are provided in Supporting Information.

\section{Antibody conjugation to microbeads and calibration procedures}

Antibodies were biotinylated using Vector Laboratories Biotin (long arm) NHS reagent and were conjugated to superavidin beads (details in Supporting Information). Six dilutions of a fluorescent secondary antibody (FSA) solution containing FITC-conjugated anti-mouse IgG were prepared to produce an immunoassay calibration curve. The highest FSA concentration was chosen to correspond approximately to that required for full surface coverage of one functionalised bead per droplet. For offchip experiments (see Supporting Information), functionalised beads were incubated with each dilution, before washing the beads and imaging them between a coverslip and a microscope slide (10 beads imaged per dilution). For on-chip experiments, FSA solutions and functionalised beads were encapsulated in droplets, using a new device for each different concentration (thereby negating any carry-over effects, or indeed non-specific binding on the channels). Fluorescence images were acquired every 30 minutes and the intensity measured for 10 beads at each time point (an unmeasured droplet was used for each acquisition).

\section{Cell culture and cell lysis protocols}

Human embryonic kidney (HEK-293) cells were transfected with a mammalian expression vector for HRas-mCitrine (plasmids were a kind gift from Philippe Bastiaens) and a human breast cancer (MCF-7) cell line expressing the fusion protein EGFP-actin and neomycin resistance was created. For on-chip lysis, cells were re-suspended in an appropriate volume of $0.6 \mathrm{M}$ D-sorbitol, to give at a final concentration of $4 \times 10^{6}$ cells $/ \mathrm{ml}$. Clarified lysate solutions prepared off-chip were used at three different PBS dilutions with 1\% v/v BSA: 1/10, 1/50 and 1/100. Details of all the transfection, harvesting and offchip cell lysis protocols are provided in Supporting Information. 


\section{Protein quantification by Western Blot}

Western blot analysis was employed to measure the HRas-mCitrine concentration in the HEK-293 lysate. Details of the Western Blot protocol are provided in Supporting Information. For quantification, the raw light intensity captured for a 5 min accumulation time using a G:Box imaging system (Syngene) was measured for each band using GeneTools software. To calculate the HRas-mCitrine concentration, the mean intensity of the bands resulting from the known quantity of GST (6.15 pMoles) was used to normalise the mean intensity obtained from the HRas-mCitrine bands.

\section{Fluorophore intensity normalisation}

To compare the acquired intensity obtained from different fluorophores, filter sets and acquisition times, an intensity normalisation algorithm was written in Matlab. In this, one dye (fluorescein isothiocyanate) was assigned as a reference and the fluorescence intensity acquired from all other dyes was normalised to it. Details of the calculations and fluorophore parameters employed are provided in Supporting Information. Effects of solution $\mathrm{pH}$ on the measured intensity were not taken into account, as emission intensity changes due to $\mathrm{pH}$ are minimal over the $\mathrm{pH}$ range used (7.4-8.0) ${ }^{29}$.

\section{Device Operation \& Immunoassay Protocol}

A schematic of the device structure and images showing the operation of the microfluidic system are shown in Fig. 1. The device comprised three inlets, one for the injection of the continuous phase (A in Fig. 1a) and two for the dispersed phase (B and C). An aqueous solution containing either a cell suspension, cell lysate or a fluorescent antibody solution was injected from inlet C. Functionalised beads in either PBS or G-actin buffer were injected from inlet B, whilst the oil-surfactant solution was injected from inlet $\mathrm{A}$. The aqueous fluid streams met at a $\mathrm{Y}$ junction (D) and flowed parallel within the emerging channel until they met a T-junction (E), where water droplets in oil (W/O) were generated (Fig. 1b). Syringe pumps (New Era Pump System Inc.) were used to control the flow rates of the continuous (1 $\mu \mathrm{L} / \mathrm{min})$ and dispersed phases $(0.5 \mu \mathrm{L} / \mathrm{min}$ for each inlet $)$, resulting in stored droplets approximately 100 $\mu \mathrm{m}$ in diameter and constricted by the depth of the channel $(50 \mu \mathrm{m})$. 
The low Reynolds number $\left(\operatorname{Re} \sim 4^{*} 10^{-3}\right)$ of the system suggests that flow is laminar and that the transport of substances from one aqueous stream to another was negligible, as shown in Fig. 1c-d. Only following droplet formation were the two aqueous solutions mixed, due to well-known convective phenomena that originate at the interface of a $\mathrm{W} / \mathrm{O}$ drop moving inside a channel ${ }^{30}$. As previously reported, bead encapsulation followed Poisson statistics ${ }^{31}$, which depends solely upon the initial concentration of suspended particles and, for the concentration used, resulted in approximately $11 \%$ of droplets formed containing one bead, with the presence of empty droplets $(85 \%)$ not limiting the analytical throughput.

When cells were injected from inlet $\mathrm{C}$, an electric field was applied to the interdigitated microelectrodes (position marked in Fig. 1a) in order to trap and permanently electroporate the cells that flowed over the electrode array. Cell trapping due to positive dielectrophoresis occurred when the dielectrophoretic force $\left(F_{D E P}=2 \pi \varepsilon_{m} r^{3} \operatorname{Re}[C M F] \nabla|\vec{E}|^{2}\right)$ experienced by the cells was able to overcome the Stokes' drag force $\left(F_{S D}=6 \pi \eta r v\right)$ on the cells (where $\varepsilon_{m}$ is the dielectric permittivity of the medium, $r$ is the cell radius, $C M F$ is the Clausius-Mossotti factor ${ }^{32}, E$ is the electric field, $\eta$ is the dynamic viscosity of the medium and $v$ is the difference between the fluid dynamic velocity of the particle and that of the medium). This was achieved by applying a $20 \mathrm{~V}$ square wave with a frequency of $1 \mathrm{MHz}$, typically resulting in cell trapping efficiencies $>95 \%$. 

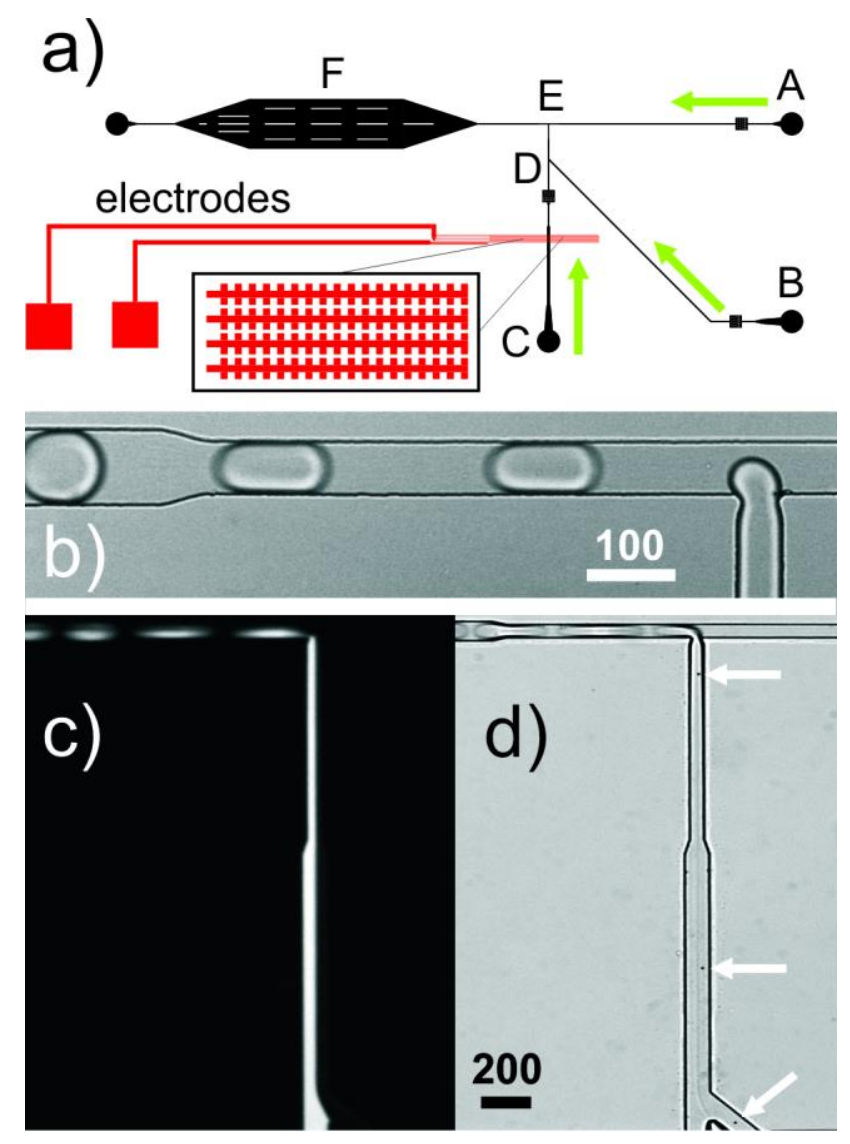

Figure 1. The device architecture: (a) Geometry of the microfluidic device. Inlet A was used to inject oil-surfactant solution (FC40 with $2 \%$ surfactants), inlet B to inject a suspension of functionalised beads and inlet $\mathrm{C}$ to inject either a calibration solution, a lysate or a cell suspension. The two aqueous phases merged at a Y junction (D) and further downstream W/O droplets were generated at a T-junction (E). Droplets were stored after the junction in a microfluidic chamber (F). Interdigitated square-shaped microelectrodes were aligned to the channel between inlet $\mathrm{C}$ and the $\mathrm{Y}$ junction to electrically lyse flowing cells; (b) Image showing droplet formation at the T-junction; (c-d) Fluorescence and bright field images showing laminar flow at the $\mathrm{Y}$-junction. The fluorescent phase flowing from inlet $\mathrm{C}$ was a FSA solution. White arrows indicate functionalised beads within the aqueous stream. Scale bars are in $\mu \mathrm{m}$.

For the conditions used ( $1 \mathrm{MHz}, 20 \mathrm{~V}, 0.6 \mathrm{M} \mathrm{D}$-sorbitol), the electric field strength was sufficient to lyse all cells captured at the electrodes (see Fig. S2 and Movie1 in Supporting Information), resulting in the release of their intracellular contents into the surrounding medium, which was subsequently 
encapsulated within droplets. Cell lysis results from the permanent electroporation of the plasma membrane ${ }^{17}$ and is caused by electrically-induced mechanical stresses. Lysis occurred over time scales in the order of hundreds of milliseconds. On average, 80 cells/min were lysed using this protocol.

Whilst cell lysate and antibody dilutions prepared off-chip were homogenous, leading to the formation of droplets containing the same number of fluorescent molecules, during on-chip cell lysis a nonuniform stream of fluorescent molecules was released into the flow, leading to a higher variability in the fluorescent contents of droplets. This was due to two factors, namely that the level of fluorescent fusion protein expression varied from cell to cell, and that the number of cells being lysed at the one time was variable - typically 1-3 cells were trapped by the electrodes at the same time (Fig. S2). For the cell suspension concentration used, we rarely observed periods in which no cells were trapped at the electrodes. Variability in the amount of cellular lysate between droplets was compensated for by averaging the signal from at least ten drops for each sample and time point.

After electro-lysis, cell fluorescence decreased to $30-50 \%$ of its original value for both cell lines used. An example of the lysis of a HEK-293 cell expressing HRas-mCitrine is shown in Fig. 2a. It would not be expected that all the intracellular molecules of interest would be released into the surrounding media, with a significant number of proteins being, for instance, membrane-associated (it is known that modification of Ras can produce a membrane-anchored form) or attached to cytoskeletal components (actin being a key component of the cytoskeleton). For the flow rates and electric fields used, cells detached from the electrodes 0.3-1 s after trapping and lysis. However, the decrease in cell fluorescence typically occurred in the first $500 \mathrm{~ms}$. When detached from the electrodes, cell debris was either absorbed onto the channel walls prior to the Y-junction or occasionally encapsulated in droplets. Cell debris did not hinder the device functionality, nor cause channel clogging, and droplets encapsulating visible cell debris were not selected for analysis.

A chamber ( $\mathrm{F}$ in Fig.1a) was used to store droplets after formation. The rate of formation was 20 droplets per second and the chamber filled in approximately 2 minutes (although droplets were allowed to flow for 5 minutes to achieve a steady condition for cell injection). After this period, the flow was 
stopped. Typically, droplet volumes decreased by $25 \%$ with respect to the initial volume after 5 hours, due to water absorption into the PDMS ${ }^{33-35}$. The storage area allowed for the collection of approximately 2000 droplets, with around 220 of these containing a single bead.

Immediately after droplet storage, bead fluorescence measurements were acquired for 10 different beads at intervals of 30 minutes. Once a bead and lysate were encapsulated in a droplet, fluorescent fusion proteins specifically bound to the antibody-conjugated bead, increasing the bead fluorescence signal with time. Transport of antigens to the bead surface was due both to convective motions that caused fluid mixing inside the droplet during droplet transport (which lasted 2-3 minutes) and to diffusion, which occurred over longer time scales when the droplets were stored inside the chamber. Convection inside travelling droplets constitutes one of the advantages offered by droplet microfluidics for obtaining rapid results.

An example of a fluorescence image from beads encapsulated in droplets stored in the microfluidic chamber is given in Fig. 2b. As described in Fig. 2c-d, the fluorescence signal ( $\Delta \mathrm{I}$ in Fig. $2 \mathrm{~d})$ was calculated as the difference between the mean value of the fluorescence intensity from the bead and the mean value of that obtained from the background (from an intensity line plot passing across the centre of each bead). Antigen concentrations significantly above saturation levels were avoided. 

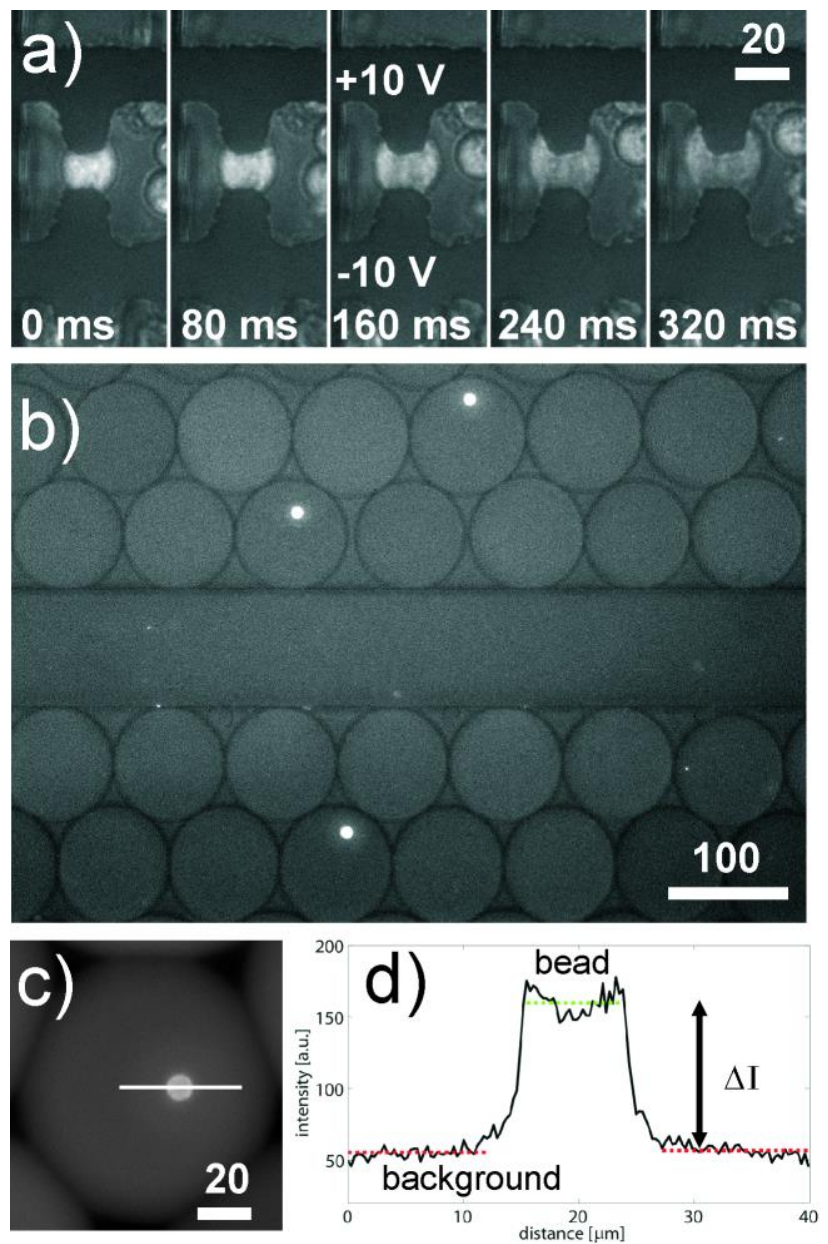

Figure 2. Fluorescence measurements of protein capture: (a) Sequence of fluorescence images of a HEK-293 cell expressing HRas-mCitrine being electrically lysed at a microelectrode. It can be seen that the cell diameter increased with time due to the effect of rupturing the plasma membrane; (b) Fluorescence image of microdroplets stored inside the chamber after a 1 hr incubation; (c-d) The method used to measure fluorescence intensity for immunoassay analysis. The white line, which runs through both the centre of the bead and the surrounding background (c), shows the position of the line plots acquired. The fluorescence intensity, $\Delta \mathrm{I}$, was calculated (d) as the mean bead intensity (green dashed line) minus the mean background intensity (red dashed line). Scale bars are in $\mu \mathrm{m}$.

\section{Results \& Discussion}

The capture and detection of antigens by antibody functionalised beads was carried out both on- and off-chip, using known concentrations of fluorescent secondary antibody calibrants, in order to determine 
the sensitivity of both droplet and conventional bead-based immunoassays. The calibration curve obtained from the on-chip experiments was later used to estimate the concentration of fluorescent fusion proteins from fluorescence measurements obtained during experiments using cells and cell lysates. The time dependence of the fluorescent measurements and the level of non-specific adsorption were also characterised.

Experiments using a cell lysate prepared off-chip were also performed, to compare the on-chip results with those obtained by Western blot analysis and to assess the performance of the immunoassay when using complex, biologically relevant samples. Finally, both HEK-293 (expressing HRas-mCitrine) and MCF-7 (expressing Actin-EGFP) cells were electrically lysed on-chip and the concentration of fluorescent fusion proteins measured and compared to previous results.

\section{Calibration protocol and non specific absorption assays}

The calibration curves in Fig. 3a show the mean values of the normalised fluorescence intensity acquired after 2 hours of incubation, as a function of FSA concentration, for both on- and off-chip experiments. As expected, the fluorescence intensity acquired from beads (coated with mouse antiHRas) encapsulated in droplets was lower than that detected from the off-chip experiments, presumably due to light scattering resulting from the multilayer emulsion system (glass-HSQ-oil-water). However, both curves have a sigmoidal shape, with the top right plateau of the sigmoid corresponding to the fluorescence from a saturated bead and the bottom left plateau stemming from the combined intrinsic fluorescence of the bead and the droplet system. From these experiments, a detection limit of $\sim 50 \mathrm{pM}$ was obtained for the immunoassay (and the $\mathrm{K}_{\mathrm{d}}$ of the antibody binding was estimated to be $50 \mathrm{nM}$ from a semilog plot of the experimental data).

The primary difference between the off- and on-chip experiments is that no washing steps could be carried out within the droplets. Thus, all proteins that were encapsulated within a droplet, but not bound to a bead, remained within the aqueous phase and the level of bead fluorescence acquired depended on the rates of mass transport and the binding kinetics of the antibody-antigen used. In all cases, it was 
therefore important to ensure that the fluorophore concentration was within the linear binding range and that sufficient time was allowed for mass transport to reach equilibrium.
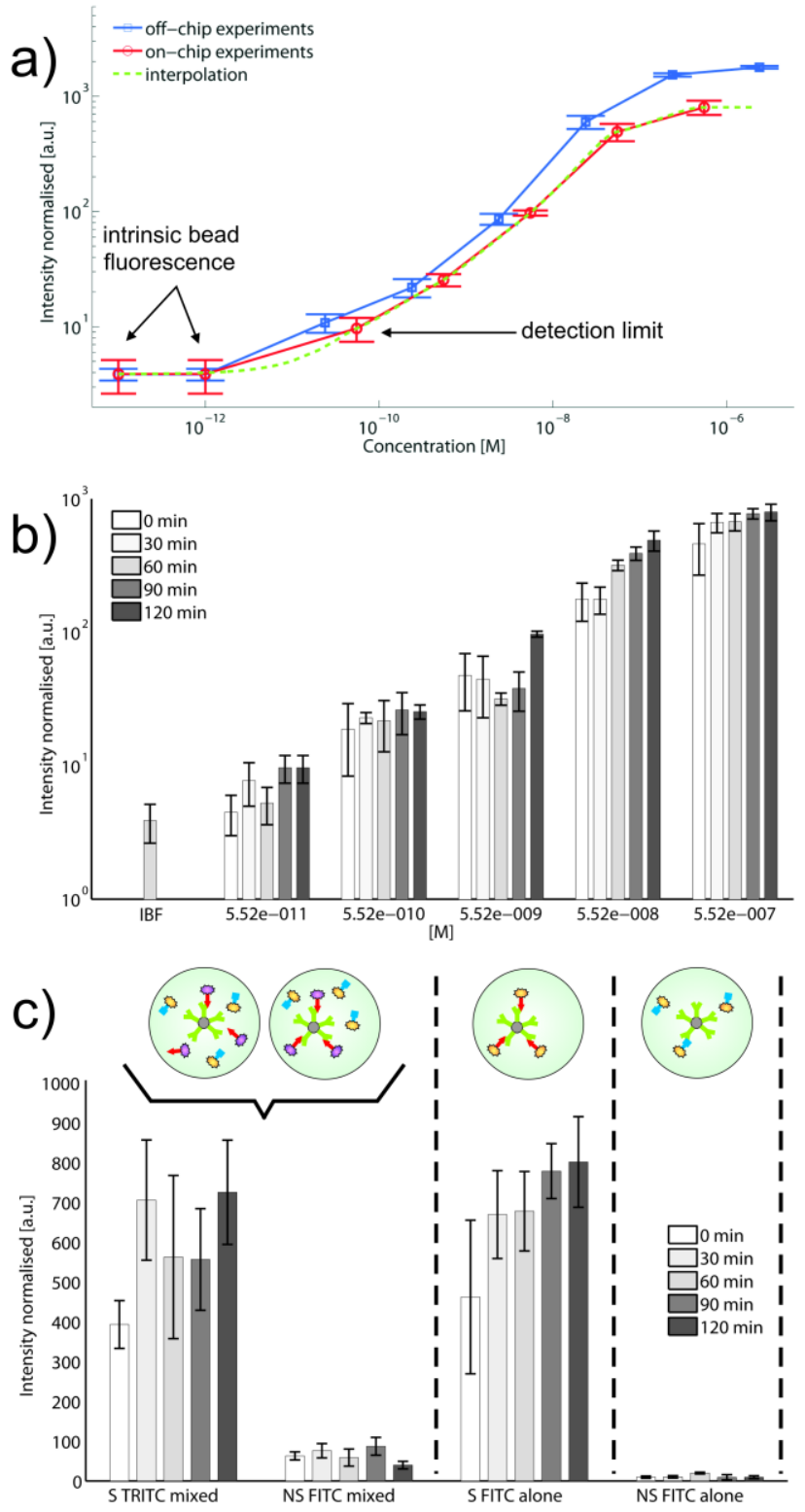

Figure 3. Calibration curves and immunoassay time response: (a) off-chip (blue) and on-chip (red) calibration curves obtained by incubating beads with several known FSA concentrations. The values correspond to the mean intensity levels acquired after 2 hours of incubation. The dashed line (green) represents a data interpolation for on-chip experiments, obtained by applying a Piecewise cubic Hermite algorithm in Matlab; (b) Time courses of the mean, normalised, fluorescence intensity levels, obtained from on-chip experiments, compared to the intrinsic fluorescence of a bead encapsulated in a droplet 
(IBF) for different FSA concentrations. Molar concentrations of FSA below approximately 50 pM could not be resolved; (c) Time dependent specific (S) vs. non-specific (NS) absorption of FSA, for $1 \mu \mathrm{M}$ solutions. The graph shows mean intensity levels obtained from droplet encapsulation of a solution containing only non-specific (anti-rabbit) antigens (right), only specific (anti-mouse) antigens (centre) and a 1:1 mixture of both specific and non-specific antigens (left).

Fig. $3 \mathrm{~b}$ shows the normalised fluorescence intensities obtained from the on-chip calibration experiments as a function of time, compared to the intrinsic bead fluorescence (IBF). For each experiment, the variation in fluorescence intensity showed a trend that increased with time until an equilibrium condition dependent upon the antibody-antigen binding kinetics was reached. It should be noted that time $t=0$ corresponds to the point that, following filling of the storage chamber, the flow was stopped. Therefore, at this stage the beads had already been incubated with the FSA solution for 2-3 min. For concentrations $>50 \mathrm{pM}$, the initial measurements showed fluorescence intensities that were significantly greater than the IBF level and increased with FSA concentration. This rapid response was largely due to convective movements within the droplets, which occurred during the transport of a droplet from the T-junction to its resting position in the chamber. Such convective motion enhanced the mixing between the two aqueous phases (the bead suspension and the FSA solution). Due to the emulsion stability, time course measurements could be taken over many hours.

To assess the level of non-specific adsorption by the functionalised beads, on-chip experiments were carried out using solutions containing positive (TRITC-conjugated or FITC-conjugated anti-mouse) and negative (FITC-conjugated anti-rabbit) secondary antibody controls. Experiments were carried out using single and mixed solutions of antibodies. The antibody concentrations were $1 \mu \mathrm{M}$ in all cases, which is approximately the concentration required for saturation of the available binding sites on the functionalised beads (determined from Fig. 3a). Time lapsed fluorescence intensity images were again acquired every 30 minutes for 2 hours. 
As shown in Fig. 3c, whether the antigens were encapsulated in droplets separately or mixed, the level of non-specific adsorption was substantially lower than specific antigen binding. Fluorescence intensity due to non-specific adsorption was estimated to be $<12 \%$ of the specific binding level obtained. Whilst the latter followed the same time-course as seen in previous experiments, the level of fluorescence intensity obtained from non-specific antigen binding remained approximately constant throughout the 2 $\mathrm{h}$ incubation period.

\section{Cell lysate and on-chip cell electro-lysis assays}

To validate our droplet-based protein assay, cellular lysates containing fluorescent fusion proteins were used. Whilst this approach was used for proof-of-concept demonstration, alternative strategies using a second labeled antibody (e.g. a sandwich assay) could be used within the system to quantify levels of endogenous proteins. Clarified cell-lysate from HEK-293 cells expressing HRas-mCitrine was prepared off-chip as described in Supporting Information. As their high concentration of detergent (1\% Triton X-100) was sufficient to cause emulsion instability, undiluted lysate solutions could not be used. Therefore, three cell lysate dilutions - 1:10, 1:50 and 1:100 lysate:PBS - were prepared and injected into the device from inlet C. These results obtained are reported in Fig. 4.

The intensity levels acquired from single anti-HRas functionalised beads encapsulated in droplets were much greater than the detection limit of the assay and showed a time dependence similar to that obtained in the calibration experiments (Fig. 4a). HRas-mCitrine concentrations were estimated by comparing the intensity levels from the cell lysate experiments with the normalised, interpolated calibration curve (Fig. 4b). This resulted in concentrations of $2.1 \mathrm{nM}$ HRas-mCitrine for the 1:100 dilution, $2.7 \mathrm{nM}$ for the 1:50 and $11 \mathrm{nM}$ for the 1:10, suggesting that the concentrations of HRas were at the low end of the linear binding range. Using an average of the 1:50 and 1:10 measurements gives an approximate concentration of $120 \mathrm{nM}$ for the undiluted lysate. 


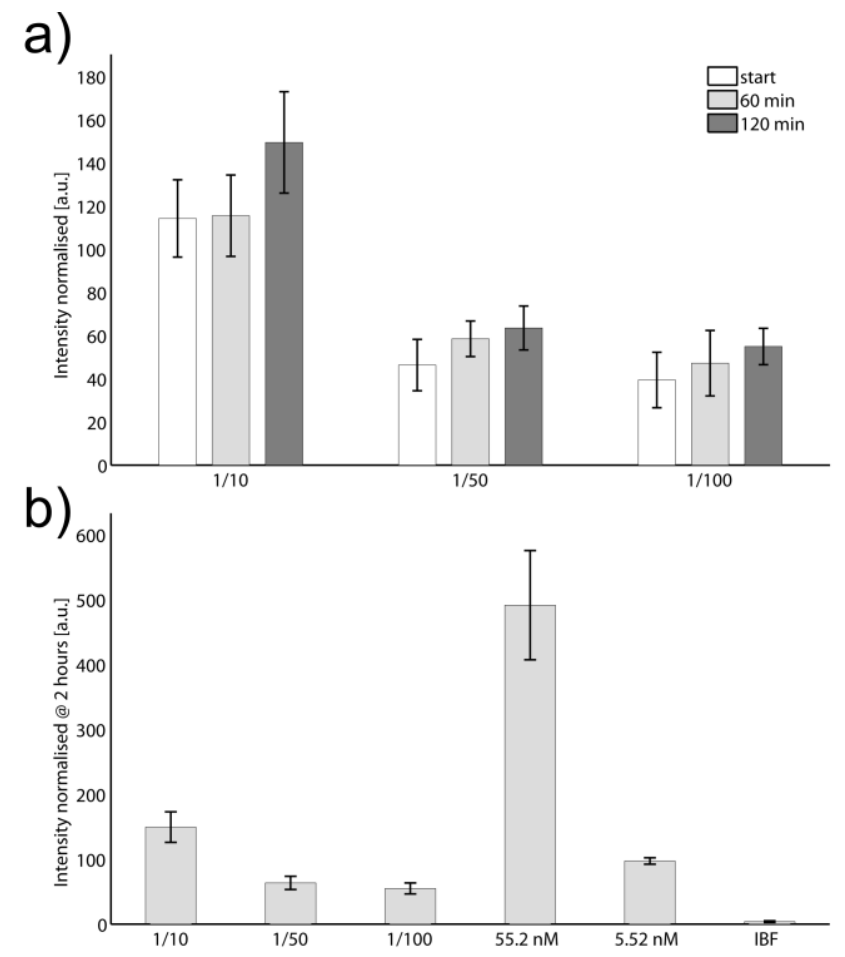

Figure 4. On-chip HEK-293 lysate experiments: (a) Time-dependent, mean, normalised fluorescence intensity obtained from 1:10, 1:50 and 1:100 lysate:PBS solutions; (b) Mean, normalised fluorescence intensity acquired after $2 \mathrm{~h}$ incubation, comparing the cell lysate dilutions to calibration experiments and to the intrinsic bead fluorescence (IBF) level. Data are representative of at least 5 experiments.

As the $K_{d}$ of the anti-HRas antibody was unknown, in order to validate the results obtained from the on-chip procedure, a series of Western Blots were performed using the same clarified cell lysate along with a protein standard (examples of the resulting blots are shown in Supporting Information). Quantification was performed by comparing the mean, background-corrected intensity of the HRas-mCitrine bands with the mean obtained from a known concentration of the protein standard (6.2 pMoles of purified glutathione $S$-transferase). The results gave a HRas-mCitrine concentration of approximately $390 \mathrm{nM}$, which corresponds well with the data obtained on-chip (differences may arise from losses due to non-specific adsorption within the device and tubing). As cell lysate solutions contain a multitude of proteins in addition to the one being detected, these results demonstrate the robustness and reliability of the on-chip immunoassay. It is also worth noting that many biological applications 
require only relative quantification, measuring either the up- or down-regulation of protein expression levels. In these cases, only relative intensity changes are required, measurement of which is straightforward using this system.
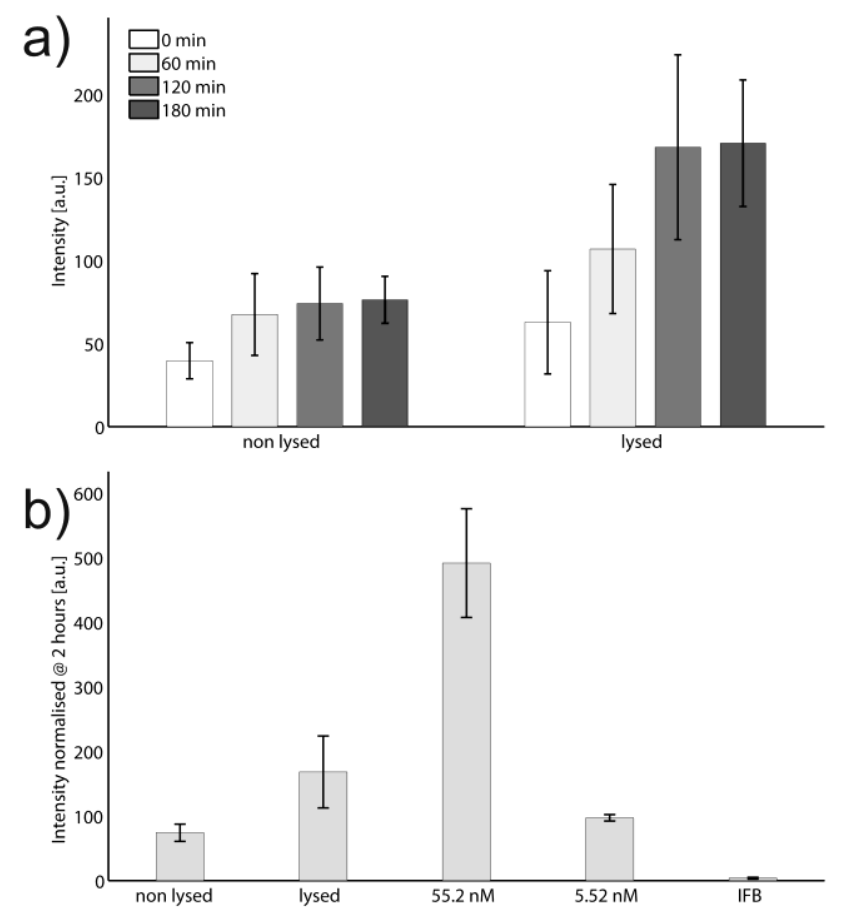

Figure 5. Detection of HRas-mCitrine in on-chip cell lysis experiments: (a) Time dependent, mean, normalised fluorescence intensity obtained for both lysed and unlysed HEK-293 cells; (b) Normalised fluorescence intensity acquired after 2 hours of incubation, comparing the on-chip cell lysis experimental data to calibration experiments and to the intrinsic bead fluorescence. Data are representative of at least 5 experiments.

HEK-293 cells expressing HRas-mCitrine were used to validate the on-chip cell lysis procedure in combination with the immunoassay for the detection of cytoplasmic proteins. Ras proteins belong the family of small GTPases and are involved in intracellular signalling pathways that regulate numerous cellular processes, including proliferation, differentiation, adhesion, migration and apoptosis ${ }^{36}$.

Typically, less than 1000 cells were injected into the chip. Cells were electrically lysed using the onchip microelectrodes (with the parameters used leading to a lysate concentration equivalent to 
approximately 0.07 cells per droplet) and the immunoassay results obtained are shown in Fig. 5. Although expression levels varied widely from cell to cell (as is normal for a population of transiently transfected cells), the majority of the HEK-293 cells expressed high levels of the HRas fusion protein. These high levels are known to be rather cytotoxic and a number of dead cells were observed in the initial cell suspension, leading to a high background fluorescence level. The cells were also susceptible to lysis under mechanical stress, preventing extensive washing. Experiments were therefore carried out with lysis electrodes both active and inactive, to enable a comparative measurement.

Fig. 5a shows the immunoassay time courses obtained. By measuring the difference in intensity obtained with the microelectrodes inactive (the non-lysed case) and with the electrodes active (and lysing the flowing cells) and comparing to the calibration curve, protein quantification was possible. Intensity values for the non-lysed case corresponded to a HRas-mCitrine concentration of $3.5 \mathrm{nM}$, whilst the value obtained when lysing the cells was $13 \mathrm{nM}$, giving a value of approximately $10 \mathrm{nM}$ for the contribution due to the cells lysed on-chip. This is in close agreement with the value from the Western Blot data: approximately 80 cells per min were lysed and 20 droplets (approximately $260 \mathrm{pL}$ ) per second produced within the device, giving on average the content of 0.066 cells per droplet. This is equivalent to approximately $2.6 \times 10^{5}$ cells per $\mathrm{mL}$, which is approximately 0.026 that of the lysate $\left(1 \times 10^{7}\right.$ cells per ml) that was measured by Western Blot to contain approximately 390nm HRas-mCitrine. A 0.026 dilution of this would therefore be in the order of 10nM.

Finally, MCF-7 cells expressing actin-EGFP were used to further validate the combined on-chip lysis and immunoassay system for the detection of cellular proteins. Actin is a globular, cytoskeletal protein found in high concentration in eukaryotic cells (can be $>100 \mu \mathrm{M}$ ) and is involved in key cellular processes, including muscle contraction, cell motility, cell division and the establishment and maintenance of cell junctions ${ }^{37}$. Actin monomers polymerise to form microfilaments within the cell, which constitute one of the three major components of the cell cytoskeleton. 

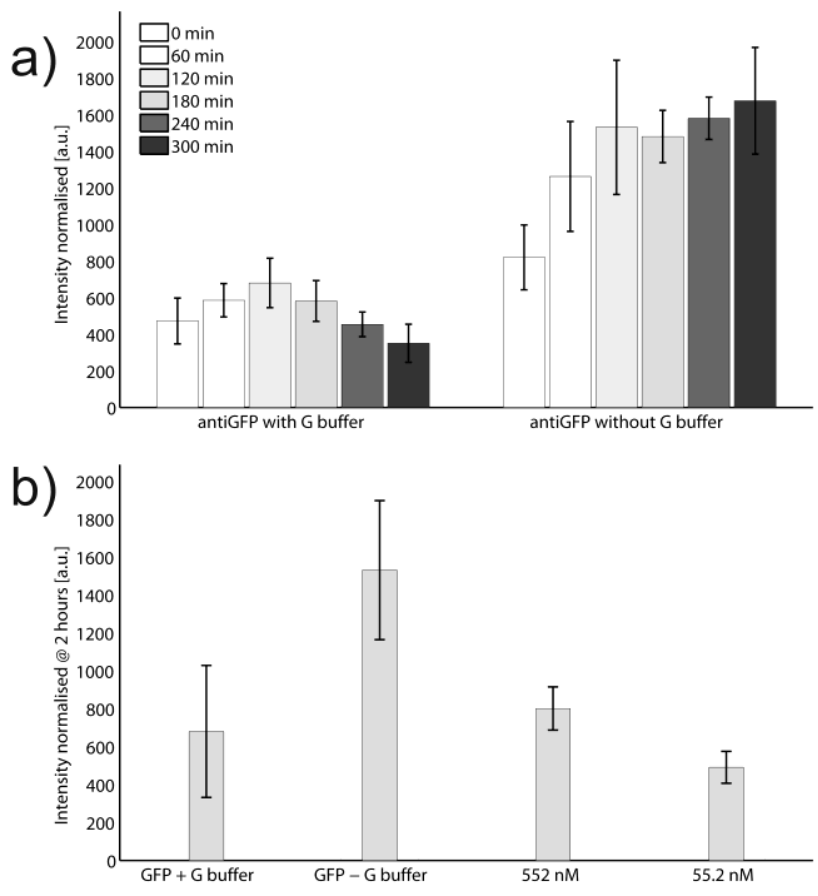

Figure 6. On-chip MCF-7 cell lysis experiments: a) Time dependent, mean normalised fluorescence intensity levels obtained from on-chip electrically lysed cells expressing actin-EGFP. Two immunoassays were performed, both using antiGFP-conjugated beads but each using a different buffer solution, either G-buffer (left) or PBS (right); (b) Mean, normalised fluorescence intensity levels acquired after $2 \mathrm{~h}$ incubation, comparing experimental data from the on-chip cell lysis experiments to calibration experiments. Data are representative of at least 5 experiments.

Actin is present both in monomeric and polymeric forms inside a cell, each form of which will clearly result in different levels of fluorescence when captured by a functionalised bead. Therefore, beads conjugated to anti-EGFP antibodies were suspended in one of two different buffer solutions prior to injection into the chip, either PBS or G-actin buffer. Whilst the former does not affect levels of polymerised actin, the latter promotes its depolymerisation. The results obtained when using these two buffer solutions, which exhibited different trends, are shown in Fig. 6. When G-actin buffer was employed, the intensity levels were significantly lower than when using PBS, confirming filament disassociation. Instead, when no action was taken to disrupt actin polymerisation, the intensity levels increased over time, reaching equilibrium after approximately $2 \mathrm{~h}$. When the electrodes were inactive 
(and therefore no cells were lysed), the level of bead fluorescence remained at the same level as the intrinisic bead fluorescence.

By comparing the results from the on-chip MCF-7 cell lysis experiments with the calibration curve, actin-EGFP concentrations could be estimated (Fig. 6b). The intensity values obtained when using Gactin buffer corresponded to the saturation of the bead, whilst the values obtained when using PBS corresponded to intensity level well above the bead saturation. In the first case, the results suggest that monomeric and small filaments of actin-EGFP were captured by the beads; whilst in the second case, longer filaments of actin-EGFP were instead bound, enabling the captured protein level to be well above that of monolayer coverage for an individual protein.

\section{Conclusions \& Perspectives}

In this work, we have presented a novel droplet microfluidic system for performing quantitative onchip immunoassays with integrated cell lysis. By taking full advantage of the efficiency and functionality of LOC devices, similar results to those obtained by Western blot were achieved in shorter times, using a reduced number of cells. Whilst lysate solutions are typically produced from several million cells, less than a thousand cells were required to obtain reliable results using the on-chip immunoassay. This is clearly valuable for the analysis of rare cell populations.

On-chip purification or washing stages were not required, greatly simplifying the device geometry and the complexity of operations. Nevertheless, droplet microfluidics can provide a library of operations that can be implemented for such purposes or for incorporating additional functionalities. For example, electrocoalescence of different droplet populations ${ }^{38}$ and subsequential splitting ${ }^{39}$ can be readily implemented within the channel network. Such operations, combined with two connected storage arrays, would enable a two-stage immunoassay that could be used for the detection of endogenous proteins. Furthermore, by exploiting both the compartmentalisation properties offered by microdroplet technology and available methods for creating a library of encoded beads, each functionalised with a different 
antibody, we envisage the development of rapid, low-volume, multiplexed immunoassays based upon this droplet microfluidic system.

ACKNOWLEDGMENTS: This work was supported by BBSRC (BB/F005024/1), RASOR (BBSRCEPSRC IRC), the DTC in Proteomics and the Royal Thai Government. The authors thank Prof. Robert Insall for helpful advice on the actin-EGFP experiments.

Supporting Information Available. Materials, setup and device fabrication details; cell culture and cell lysis protocols; fluorophore normalization; Western blot analysis, Figure S1-S2 and Movie1. This material is available free of charge via the Internet at http://pubs.acs.org.

\section{REFERENCES}

(1) Renart, J.; Reiser, J.; Stark, G. R. Proc. Natl. Acad. Sci. U. S. A. 1979, 76, 3116-3120.

(2) Engvall, E.; Perlmann, P. Immunochemistry 1971, 8, 871-\&.

(3) Delehanty, J. B.; Ligler, F. S. Anal. Chem. 2002, 74, 5681-5687.

(4) MacBeath, G. Nat. Genet. 2002, 32, 526-532.

(5) Templin, M. F.; Stoll, D.; Schrenk, M.; Traub, P. C.; Vohringer, C. F.; Joos, T. O. Trends Biotechnol. 2002, 20, 160-166.

(6) Blicharz, T. M.; Siqueira, W. L.; Helmerhorst, E. J.; Oppenheim, F. G.; Wexler, P. J.; Little, F. d. r. F.; Walt, D. R. Anal. Chem. 2009, 81, 2106-2114.

(7) Sivagnanam, V.; Song, B.; Vandevyver, C.; Gijs, M. A. M. Anal. Chem. 2009, 81, 6509-6515.

(8) Nedelkov, D.; Kiernan, U. A.; Niederkofler, E. E.; Tubbs, K. A.; Nelson, R. W. Mol. Cell. Proteomics 2006, 5, 1811-1818.

(9) Mattoussi, H.; Mauro, J. M.; Goldman, E. R.; Anderson, G. P.; Sundar, V. C.; Mikulec, F. V.; Bawendi, M. G. J. Am. Chem. Soc. 2000, 122, 12142-12150.

(10) Zare, R. N.; Kim, S. In Annu. Rev. Biomed. Eng., 2010; Vol. 12, pp 187-201.

(11) Huh, D.; Matthews, B. D.; Mammoto, A.; Montoya-Zavala, M.; Hsin, H. Y.; Ingber, D. E. Science 2010, 328, 1662-1668.

(12) Beebe, D. J.; Mensing, G. A.; Walker, G. M. In Annu. Rev. Biomed. Eng., 2002; Vol. 4, pp 261286.

(13) El-Ali, J.; Sorger, P. K.; Jensen, K. F. Nature 2006, 442, 403-411.

(14) Weibel, D. B.; DiLuzio, W. R.; Whitesides, G. M. Nat. Rev. Microbiol. 2007, 5, 209-218.

(15) Ng, A. H. C.; Uddayasankar, U.; Wheeler, A. R. Anal. Bioanal. Chem. 2010, 397, 991-1007.

(16) Liu, W. S.; Chen, D. L.; Du, W. B.; Nichols, K. P.; Ismagilov, R. F. Anal. Chem. 2010, 82, 32763282.

(17) Florescu, O.; Wang, K.; Au, P.; Tang, J.; Harris, E.; Beatty, P. R.; Boser, B. E. J. Appl. Phys. 2010, 107. 
(18) Sedgwick, H.; Caron, F.; Monaghan, P. B.; Kolch, W.; Cooper, J. M. J. R. Soc. Interface 2008, 5, S123-S130.

(19) Yang, S. Y.; Lien, K. Y.; Huang, K. J.; Lei, H. Y.; Lee, G. B. Biosens. Bioelectron. 2008, 24, 855-862.

(20) Jin, G. B.; Unfricht, D. W.; Fernandez, S. M.; Lynes, M. A. Biosens. Bioelectron. 2006, 22, 200206.

(21) Sasso, L. A.; Undar, A.; Zahn, J. D. Microfluid. Nanofluid. 2010, 9, 253-265.

(22) Lim, C. T.; Zhang, Y. Biosens. Bioelectron. 2007, 22, 1197-1204.

(23) Theberge, A. B.; Courtois, F.; Schaerli, Y.; Fischlechner, M.; Abell, C.; Hollfelder, F.; Huck, W. T. S. Angewandte Chemie-International Edition 2010, 49, 5846-5868.

(24) Zagnoni, M.; Cooper, J. M. Lab. Chip 2010, 10, 3069-3073.

(25) Christopher, G. F.; Anna, S. L. Journal of Physics D-Applied Physics 2007, 40, R319-R336.

(26) He, M. Y.; Edgar, J. S.; Jeffries, G. D. M.; Lorenz, R. M.; Shelby, J. P.; Chiu, D. T. Anal. Chem. 2005, 77, 1539-1544.

(27) Joensson, H. N.; Samuels, M. L.; Brouzes, E. R.; Medkova, M.; Uhlen, M.; Link, D. R.; Andersson-Svahn, H. Angewandte Chemie-International Edition 2009, 48, 2518-2521.

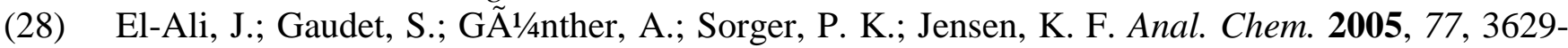
3636.

(29) Haupts, U.; Maiti, S.; Schwille, P.; Webb, W. W. Proc. Natl. Acad. Sci. U. S. A. 1998, 95, 13573-13578.

(30) Hodges, S. R.; Jensen, O. E.; Rallison, J. M. J. Fluid Mech. 2004, 501, 279-301.

(31) Di Carlo, D.; Irimia, D.; Tompkins, R. G.; Toner, M. Proc. Natl. Acad. Sci. U. S. A. 2007, 104, 18892-18897.

(32) Pethig, R. Biomicrofluidics 2010, 4.

(33) Huebner, A.; Bratton, D.; Whyte, G.; Yang, M.; deMello, A. J.; Abell, C.; Hollfelder, F. Lab. Chip 2009, 9, 692-698.

(34) Courtois, F.; Olguin, L. F.; Whyte, G.; Theberge, A. B.; Huck, W. T. S.; Hollfelder, F.; Abell, C. Anal. Chem. 2009, 81, 3008-3016.

(35) Shim, J. U.; Olguin, L. F.; Whyte, G.; Scott, D.; Babtie, A.; Abell, C.; Huck, W. T. S.; Hollfelder, F. J. Am. Chem. Soc. 2009, 131, 15251-15256.

(36) Peyker, A.; Rocks, O.; Bastiaens, P. I. H. Chembiochem 2005, 6, 78-85.

(37) Pantaloni, D.; Le Clainche, C.; Carlier, M. F. Science 2001, 292, 1502-1506.

(38) Zagnoni, M.; Le Lain, G.; Cooper, J. M. Langmuir 2010, 26, 14443-14449.

(39) Link, D. R.; Anna, S. L.; Weitz, D. A.; Stone, H. A. Phys. Rev. Lett. 2004, 92. 
SYNOPSIS TOC
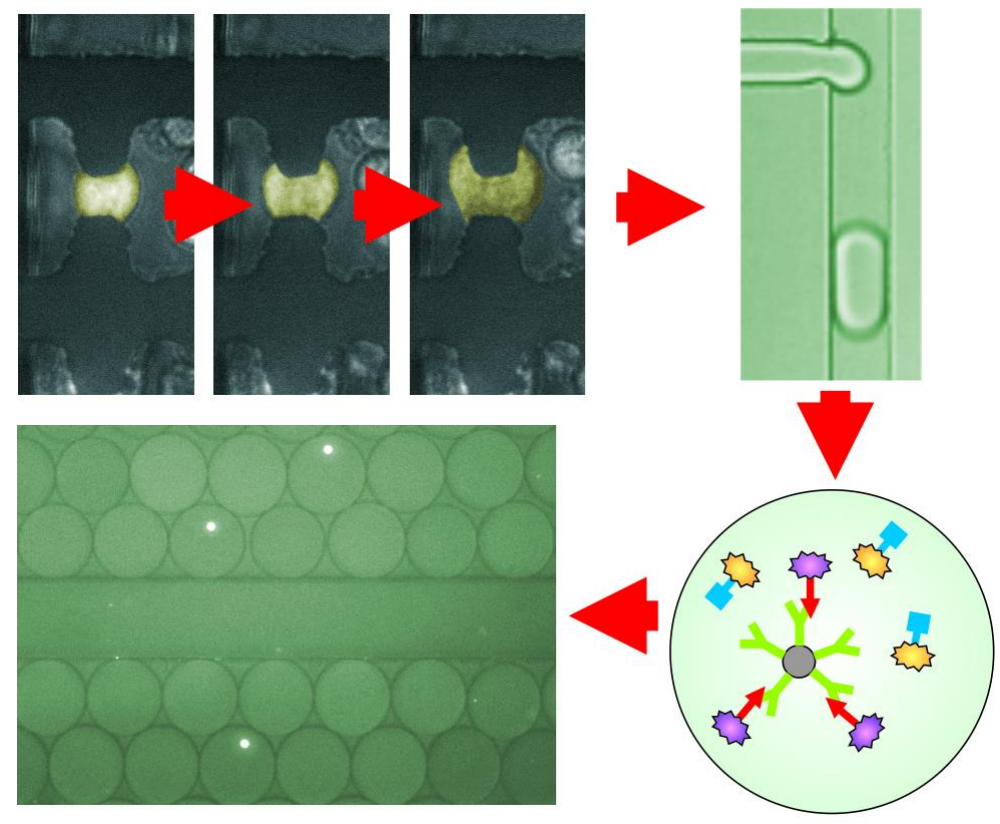